\title{
Minimal Complete Primitives for Secure Multi-Party Computation*
}

\author{
Matthias Fitzi \\ Department of Computer Science, \\ University of California, \\ Davis, CA 95616, U.S.A. \\ fitzi@cs.ucdavis.edu \\ Juan A. Garay \\ Bell Labs - Lucent Technologies, \\ 600 Mountain Ave., \\ Murray Hill, NJ 07974, U.S.A. \\ garay@research.bell-labs.com \\ Ueli Maurer \\ Department of Computer Science, ETH, \\ CH-8096 Zurich, Switzerland \\ maurer@inf.ethz.ch \\ Rafail Ostrovsky \\ Telcordia Technologies Inc., \\ 445 South Street, \\ Morristown, NJ 07960-6438, U.S.A. \\ rafail@ research.telcordia.com \\ Communicated by Oded Goldreich
}

Received October 2001 and revised June 2003

Online publication 27 April 2004

\begin{abstract}
The study of minimal cryptographic primitives needed to implement secure computation among two or more players is a fundamental question in cryptography. The issue of complete primitives for the case of two players has been thoroughly studied. However, in the multi-party setting, when there are $n>2$ players and $t$ of them are corrupted, the question of what are the simplest complete primitives remained open for $t \geq n / 3$. (A primitive is called complete if any computation can be carried out by the players having access only to the primitive and local computation.)

In this paper we consider this question, and introduce complete primitives of minimal cardinality for secure multi-party computation. The cardinality issue (number of
\end{abstract}

\footnotetext{
* A preliminary version of this paper appeared in Advances in Cryptology - CRYPTO '01, LNCS 2139,
} Springer-Verlag, pp. 80-100, Santa Barbara, CA, August 2001. 
players accessing the primitive) is essential in settings where primitives are implemented by some other means, and the simpler the primitive the easier it is to realize. We show that our primitives are complete and of minimal cardinality possible for most cases.

Key words. Multi-party computation, Secure function evaluation, Unconditional security, Complete functions, Oblivious cast.

\section{Introduction}

In (general) secure multi-party computation, $n$ parties ("players") attempt to compute multi-party functionalities in the presence of an adversary who may corrupt up to $t$ of them. The study of secure multi-party computation is important both from a theoretical and a practical point of view. It not only establishes what is possible to compute and what is not in multi-party environments, but may sometimes yield techniques and components that can be used in application-specific systems [Go1].

Traditionally, the models for secure multi-party computation have assumed that certain communication "resources" (primitives) are available to the players. Well-known instances include pairwise private channels [BGW], [CCD], a broadcast channel [RB], and, more recently, a "mini-cast" primitive, which is available to every triple of players $[\mathrm{FM}]$. In this paper, and with respect to the strongest, active adversary, we initiate the study of minimal complete primitives for secure multi-party computation from the point of view of the cardinality of the primitive-i.e., the number of players accessing it. A primitive is called complete if any computation can be carried out by the players having access (only) to the primitive and local computation. A primitive is called minimal if any primitive involving fewer players is not complete.

For $n$ players, $t$ of which might be corrupted, the question is well understood for $t<n / 3$. In this paper we consider this question for $t \geq n / 3$. We show that in fact there are three interesting "regions" for $t: t<n / 3, n / 3 \leq t<n / 2$, and $n / 2 \leq t<n$, and present, for each region, minimal complete primitives for $t$-resilient unconditional multi-party computation.

\subsection{Motivation}

There are three main motivations for this work. First, determining the minimal necessary primitives for secure multi-party computation, for various adversary models, is of fundamental theoretical interest. Second, minimizing the number of players involved in a primitive is important because the lower the primitive's cardinality the easier it might be to realize it by exploiting some physical phenomenon (e.g., quantum entanglement). For instance, broadcast among three players [FM] appears to be a more realistic primitive than Internet-scale broadcast. Third, investigating information-theoretic reductions of multi-party computation to a certain primitive is of interest, even if the implementation of this primitive is based on cryptographic assumptions, for the following reasons:

- The (cryptographic) security proof of a protocol for a complex task is typically easier when the analysis can be broken up into a global information-theoretic argument and a "local" cryptographic argument.

- Information-theoretic multi-party protocols are typically more efficient (though less resilient) than their cryptographic counterparts, and hence the described design approach may lead to the most efficient multi-party protocols. 


\subsection{Prior and Related Work}

Secure multi-party computation. Secure multi-party computation has been actively studied since the statement of the problem by Yao in [Ya]. For the standard model with secure pairwise channels between the players, the first general solution of the problem was given by Goldreich et al. [GMW] with respect to computational security. Ben-Or et al. [BGW] and Chaum et al. [CCD] constructed the first general protocols with unconditional security. Additionally, it was proven in [BGW] that unconditionally secure multi-party computation was possible if and only if less than half (one-third) of the players are corrupted passively (actively).

For the model where, in addition to the pairwise secure channels, a global broadcast channel is available, Rabin and Ben-Or [RB] constructed a protocol that tolerates (less than) half of the players being actively corrupted. Their solution is not perfect, as it carries a small probability of error. However, it was later shown by Dolev et al. [DDWY] that this is unavoidable for the case $t \geq\lceil n / 3\rceil$ (and the assumed communication primitives), as there exist problems with no error-free solutions in this setting. Fitzi and Maurer [FM] recently proved that, instead of global broadcast, broadcast among three players is sufficient in order to achieve unconditionally secure multi-party computation for $t<n / 2$.

Complete primitives. Another line of research deals with the completeness of primitives available to the players. Kilian [Ki1] proved that oblivious transfer (OT) [Ra] is complete for two-party computation in the presence of an active adversary. A complete characterization of complete functions for two-party computation, for both active and passive adversaries, was given in [Ki3] based on [Ki2] and results by Beimel et al. [BMM]. These results are stated with respect to asymmetric multi-party computation in the sense that the result of the function is provided to one single (predefined) player.

A first generalization of completeness results to the more general $n$-party case was made by Kilian et al. [KMO], [KKMO], who characterized all complete boolean functions for multi-party computation secure against a passive adversary that corrupts any number of players.

With the noted exception of Goldreich's treatment of reductions in [Go2], previous work on complete primitives typically assumes that the cardinality of the primitive is the same as the number of players involved in the computation. ${ }^{1}$ In contrast, in this paper we are concerned with the minimal cardinality of complete primitives for multi-party computation.

\subsection{Contributions of This Paper}

In this paper, for any primitive cardinality $k, 2 \leq k \leq n$, we give upper and lower bounds on $t$ such that there is a complete primitive $g_{k}$ for multi-party computation secure against an active adversary that may corrupt up to $t$ of the players. To our knowledge, this is

\footnotetext{
${ }^{1}$ Implicitly, though, Kilian and coworkers [KMO], [KKMO] transform calls to a cardinality $n$ primitive into pairwise OT invocations. Fitzi and Maurer's work on achieving global broadcast (multi-party computation) based on 2-cast and authenticated (resp., secure) channels can also be viewed as a step towards minimizing the primitives' cardinality.
} 
the first time that the power of the cardinality of cryptographic primitives-and their minimality-is rigorously studied.

When $k=2$, it is well known that secure pairwise channels (or, more generally, OT) are enough (complete) for $t<n / 3$, as follows from [BGW], [CCD], and [Ki1]. We show that, for $n>2$, no primitive of cardinality 2 can go above this resiliency bound, including primitives that are complete for two-party computation. Specifically, we show that there is a problem, namely, broadcast (aka Byzantine agreement) [PSL], that cannot be solved in a model where players are connected by " $g_{2}$ channels" for any two-party primitive $g_{2}$.

The case $k=3$ is of special interest. We introduce two primitives: oblivious cast, a natural generalization of oblivious transfer to the three-party case, ${ }^{2}$ and converge cast, a primitive that is related to the anonymous channel of [Ch], and show that they are complete for $t<n / 2$. In light of the impossibility result for $k=2$, these primitives are also minimal. We also show that no primitive of cardinality 3 can be complete for $t \geq n / 2$.

For the case $k=n$ we introduce another primitive, which we call the universal black box (UBB), and show that it is complete for arbitrary resiliency $(t<n)$. This primitive has interesting implications for computations involving a trusted third party (TTP), in that it enables oblivious TTPs, i.e., trusted parties which do not require any prior knowledge of the function to be computed by the players-even if a majority of the players is corrupted. The UBB is also minimal, since we also show that no primitive of cardinality $n-1$ can be complete for $t<n$, by showing that broadcast tolerating that many faults cannot be achieved by any $(n-1)$-cardinality primitive. The results mentioned so far are summarized in Table 1.

Multicast and "convergecast," with a single sender and a single recipient, respectively, constitute two natural communication models. We also show that no primitive of this kind (i.e., a primitive with one input or one output)—even of full cardinality — can achieve more than $t<n / 2$, and therefore be more powerful than our primitives of cardinality 3. In other words, with respect to these primitive types, Table 1 "collapses" to two equivalence classes: $k=2$ and $3 \leq k \leq n$. For these impossibility proofs, we use

Table 1. Complete primitives of cardinality $k$.

\begin{tabular}{ccccc}
\hline \multirow{2}{*}{$\begin{array}{c}\text { Primitive } \\
\text { cardinality }\end{array}$} & Efficient reduction & Impossibility & Primitive* & $\begin{array}{c}\text { Number of } \\
\text { instances }\end{array}$ \\
\cline { 2 - 3 }$k=2$ & $t<n / 3$ & $t \geq n / 3$ & $\mathrm{SC}_{2}$ & $2\left(\begin{array}{l}n \\
2\end{array}\right)$ \\
$k=3$ & $t<n / 2$ & $t \geq n / 2$ & $\mathrm{OC}_{3} / \mathrm{CC}_{3}$ & $3\left(\begin{array}{c}n \\
3\end{array}\right)$ \\
$4 \leq k \leq n-1$ & $t<n / 2$ & $t \geq n-2$ & $\mathrm{OC}_{3} / \mathrm{CC}_{3}$ & $3\left(\begin{array}{c}n \\
3\end{array}\right)$ \\
$k=n$ & $t<n$ & - & $\mathrm{UBB}_{n}$ & 1 \\
\hline
\end{tabular}

${ }^{*} \mathrm{SC}_{2}$ : secure channel; $\mathrm{OC}_{3}$ : oblivious cast; $\mathrm{CC}_{3}$ : converge cast; $\mathrm{UBB}_{n}$ : universal black box. $\mathrm{OC}_{3}, \mathrm{CC}_{3}$, and $\mathrm{UBB}_{n}$ are primitives introduced in this paper.

\footnotetext{
${ }^{2}$ Independently (but previously), a similar primivite called "Oblivious Multicast" was introduced by Blaze in [B1].
} 
reductions to problems in the standard model of secure point-to-point channels that are known to be unsolvable.

All the primitives we present allow for efficient secure multi-party computation.

\subsection{Organization of the Paper}

We start in Section 2 with definitions of and notation for primitives of arbitrary cardinality, the security model we use, and the notion of reducibility and completeness for functionalities and primitives of different cardinality. Section 3 contains the reductions of multi-party computation to primitives of the various cardinalities. In Section 3.1 we revisit the case of cardinality 2 with a tight bound of $t<n / 3$. In Section 3.2 we introduce the two new primitives of cardinality 3 , and show that they are complete for $t<n / 2$, which is a tight bound as it follows from Section 4; we also show in this section how other primitives of cardinality 3 can be designed in such a way that the analysis required by the reductions is less involved. In Section 3.3 we give a primitive of full cardinality $(k=n)$ that is complete for $t<n$. Section 4 contains the impossibility results, i.e., lower bounds on the required number of correct players for multi-party computation with primitives of cardinality $2 \leq k \leq n-1$, together with the proofs that primitives that are single-input or single-output cannot be complete for $t \geq\lceil n / 2\rceil$. We conclude in Section 5 with open problems and directions for further research. In some of our proofs we make use of Chernoff bounds; we include a succint description in the Appendix for convenience.

\section{Model and Definitions}

In this paper we focus on secure function evaluation (SFE) [Ya] by a set $P$ of $n$ players, where each player $p_{i}$ has an input value $x_{i}$ and obtains an output value $f_{i}\left(x_{1}, x_{2}, \ldots, x_{n}\right)$, for a (probabilistic) function $f_{i}$. We are interested in unconditional security against an active adversary who may corrupt up to $t$ of the players; i.e., the adversary may make the corrupted players deviate from the protocol in an arbitrarily malicious way, and no assumptions are made about his computational power.

In contrast to the treatment of two-party computation (e.g., [Ki2], [Ki3], and [BMM]), where only one predefined player receives the final result of the computation, our model allows every player to receive his own (in general, different) result—which corresponds to the general notion of multi-party computation in [Ya], [CCD], and [BGW]. Similarly, our definition of a primitive, as given in the next paragraph, also allows every involved player to provide an input and get an output, as opposed to just one player. Nonetheless, our constructions apply to the former model as well since for each of our complete multiple-output primitives there is also a single-output primitive that is complete with respect to single-output SFE.

\subsection{Primitives of Arbitrary Cardinality}

Our communication model is based on ideal primitives that can be accessed by $k$ players, $2 \leq k \leq n$, implementing the secure computation of some $k$-ary, possibly probabilistic function; $k$ is called the cardinality of the primitive. Besides this primitive, no other means of communication is assumed among the players. 
We view primitives as "black boxes" in the sense that all implementation details are hidden from the players. Depending on the function being implemented, of the $k$ players accessing the primitive one or more may secretly enter an input to it, and one or more may secretly receive the value(s) of the function.

We use $g_{k}[i, j]$ to denote the primitive implementing $k$-ary function $g$, in which $i \leq k$ players provide an input, and where $j \leq k$ players receive the output of the function. ${ }^{3}$ We call $[i, j]$ the type of the primitive. We drop the type when clear from the context. We focus on the following types: $[1, \ell],[\ell, 1]$, and $[k, k]$ where $1 \leq \ell \leq k^{4}$

Note that a primitive of a given cardinality can always be simulated (when applicable) by the same primitive with a larger cardinality by "cutting" some of the "wires," or connecting them to the same player. More formally, the following domination relation exists: let $\left(k^{\prime}, i^{\prime}, j^{\prime}\right) \supseteq(k, i, j)$ (meaning $k^{\prime} \geq k, i^{\prime} \geq i$ and $j^{\prime} \geq j$ ); then for every primitive $g_{k}[i, j]$ there exists a primitive $g_{k^{\prime}}^{\prime}\left[i^{\prime}, j^{\prime}\right]$ that is as powerful as $g_{k}[i, j]$.

We assume that every subset $S \subset P$ of $k$ players shares $k$ ! instances of the primitiveone for each permutation of the players; thus, we assume $\left(\begin{array}{l}n \\ k\end{array}\right) k$ ! instances of the primitive in total. However, we will show that there is always a (minimal complete) primitive such that, overall, polynomially many instances (specifically, less than $n^{3}$ ) of the primitive are sufficient.

\subsection{Security Model}

Several formal definitions of secure function evaluation exist (e.g., [Be], [Ca], [Go2], $[\mathrm{GL}]$, and $[\mathrm{MR}])$. The process is assumed to be synchronous, a fact that simplifies the task of reasoning about security. In [Ca] (and in a nutshell), the computation to be performed among the $n$ players is specified with respect to an incorruptible trusted party $\tau$ who interacts securely with the players. For the special case of STE where a function on the players' inputs is to be computed and revealed, such a process can be defined by the players first secretly handing their inputs to $\tau, \tau$ computing the output corresponding to the (possibly probabilistic) function, and then handing it back to the players. Such a protocol among $P \cup\{\tau\}$ is called an ideal process.

Of course, the goal of multi-party computation is to perform the same task without the need for a trusted party; thus, a multi-party computation protocol for evaluating a function is called secure if it emulates the ideal evaluation process of the function, i.e., if for every strategy of the adversary in the real protocol there is a corresponding adversary strategy that, with similar cost, achieves the same effect in the ideal process. In particular, this means that whenever the ideal process satisfies some consistency or privacy property with respect to the players (e.g., privately computes some specific function on the players' inputs), then the secure protocol also satisfies them. This notion of security can then be refined further by distinguishing among the different types of similarities between the global outputs in both the ideal and real-life computations. We

\footnotetext{
${ }^{3}$ A complete specification of the primitive should include additional aspects, such as which $i(j)$ out of the $k$ players provide an input (resp., receive an output), etc., but the simpler notation will be expressive enough for the primitives we consider.

${ }^{4}$ Note that, for $n \geq 2$, no primitive of type $[0, *]$ or $[*, 0]$ can be complete and thus these cases are ignored. For the same reason, in the case of $[1,1]$, we always ignore the "reflexive" case (same player providing input and receiving the output).
} 
are interested in unconditional security, which is obtained by requiring that these output distributions be (statistically) indistinguishable, except for a negligible function of the security parameter, independently of the adversary's computational power. See $[\mathrm{Ca}]$ for further details.

\subsection{Reducibility and Completeness}

A main theme in this paper is that of reductions "across" cardinalities. The notion of reduction generalizes to the case of computation of an $n$-ary function ( $n$-player protocol) ${ }^{5}$ invoking another $k$-ary function (primitive of cardinality $k$, resp.), with $k \leq n$, in a natural way [Go2]:

Definition 1 (Reductions). An $n$-player protocol unconditionally reduces $f_{n}$ to $g_{k}$ for a given $t<n$, if it computes $f_{n}$ unconditionally $t$-securely just by black-box calls to $g_{k}$ and local computation. In such a case we say that $f_{n}$ unconditionally reduces (for short, reduces) to $g_{k}$ for that $t .6$

The notion of completeness also generalizes to the different cardinality setting in a natural way: if $g_{k}$ is complete one can use $g_{k}$ to perform secure $n$-party computation. More formally:

Definition 2 (Completeness). We say a primitive $g_{k}$ is unconditionally complete (for short, complete) for a given $t<n$, if every $n$-ary function unconditionally reduces to $g_{k}$ (for the same $t$ ).

Typically, the reduction step is applied more than once, by reducing a primitive already known to be complete to another, perhaps simpler, primitive. For example, this is the case in the two-party case, where protocols are given that implement oblivious transfer using a different primitive (see, e.g., [Ki3]). This is also the approach we follow in this paper, by showing how to implement, using our primitives, the "resources" that are known to be required for SFE.

Furthermore, all our reductions will be unconditionally secure in a way that the simulation can fail with some negligible probability, but, in the non-failure case, it perfectly provides the desired functionality; i.e., compared with an ideal implementation of the functionality, the reductions leak no additional information and provide perfect correctness. Hence, by estimating the overall error probability of the complete reduction from the given SFE problem to the complete primitive as the probability that at least one single implementation of a reduction step fails, we actually get an upper bound on the probability that the whole protocol does not provide perfect security. Since our reductions keep this probability negligibly small, we achieve unconditional security according to the definition above.

\footnotetext{
${ }^{5}$ (Local) Computation can, of course, be randomized, and the requirement is that the function's output is produced with high probability.

${ }^{6}$ Note that the definition of reduction also admits the opposite direction, i.e., from smaller cardinality to larger cardinality. Occasionally in our constructions we will also use this direction (for example, by implementing secure pairwise channels using a three-player primitive).
} 
Finally, we note that all our reductions are efficient, i.e., polynomial in $n$ and a security parameter $\sigma$ such that the overall error probability is smaller than $2^{-\sigma}$.

\section{Complete Primitives}

\subsection{Primitives of Cardinality 2}

It is well known that secure channels $\left(\mathrm{SC}_{2}\right)$ are sufficient for unconditional SFE [BGW], [CCD] with $t<n / 3$. That is, in our parlance:

Proposition 3.1. For any $n \geq 2$, there is a primitive of cardinality 2 , the secure channel, that is complete for $t<n / 3$.

Since we are assuming that every permutation of the players share a primitive, the type of a secure channel is [1,1]; hence, for $t<n / 3$, the complete primitive is of the weakest type. For $n>2$, no primitive of cardinality 2 can be complete for $t \geq\lceil n / 3\rceil$ (Corollary 4.2), therefore this bound is tight.

\subsection{Primitives of Cardinality 3}

Evidently, a primitive $g_{3}[1,1]$ is equivalent to $g_{2}[1,1]$ since in $g_{3}$ one of the players neither provides an input nor receives an output. Hence, in this section we consider primitives (of cardinality 3 ) of type different from [1, 1]. In fact, it turns out that either two inputs (and single output) or two outputs (and single input) is sufficient. For each type we introduce a primitive and show it to be complete for $t<n / 2$. Moreover, we show (in Section 4) that no primitive of cardinality 3 can be complete for $t \geq\lceil n / 2\rceil$.

The approach to show that a primitive is complete is as follows. It follows from [RB] and $\left[\mathrm{CDD}^{+}\right]$that pairwise secure channels and a global broadcast channel are sufficient for SFE secure against $t<n / 2$ active corruptions. Hence, it is sufficient to show that the primitives introduced in this section imply both, unconditionally secure pairwise channels and global broadcast.

The first primitive we introduce, oblivious cast, is of type [1,2], and a generalization of oblivious transfer to the multi-receiver (in our case, two) setting: a sender sends his input bit to two receivers, who get it with probability $\frac{1}{2} .^{7}$ The second primitive we introduce, converge cast, with two senders and one receiver (i.e., of type $[2,1]$ ), is the converse of oblivious cast: each sender sends a value, and the receiver gets one value or the other with probability $\frac{1}{2}$. Thus, converge cast can be viewed as a three-party version of Chaum's "anonymous channel" [Ch].

The main reasons for considering these two primitives of cardinality 3 are that

1. they constitute natural generalizations of existing primitives to a multi-party ("network") setting, and

2. their definition is simple and symmetric (e.g., the value from each of the senders in converge cast gets through with $\frac{1}{2}$ probability); this not only makes them easy to

\footnotetext{
${ }^{7}$ See $[\mathrm{B} 1]$ for a specification for the general case $-k$ receivers out of $n$.
} 
state, but also perhaps more amenable for realization by some other (e.g., physical) means.

This, however, comes at a price, since as we describe in Section 3.2.3, other, less "natural," primitives can be designed in such a way that the implementation of the resources needed for SFE is easy, and which do not have probabilities of error. However, minimality, in particular, in the size of the input/output domains, is yet another desirable property for these primitives, of both practical and theoretical interest, and the simpler-to-analyze primitive presented there requires larger value domains than oblivious cast and converge cast. Moreover, this primitive is not symmetric. Thus, simpler analysis trades off naturally with symmetry and minimality (see Section 3.2.3).

\subsubsection{Type [1,2] Primitives: Oblivious Cast}

Definition 3. Oblivious cast $\left(\mathrm{OC}_{3}\right)$ is a primitive among three players: a sender $s$ who sends a bit $b \in\{0,1\}$ and two recipients $r_{0}$ and $r_{1}$, such that the following conditions are satisfied:

(1) The bit $b$ is received by exactly one of the recipients, $r_{0}$ or $r_{1}$, each with probability $\frac{1}{2}$.

(2) While both recipients learn who got the bit, the other recipient gets no information about $b$. In case there are other players (apart from $s, r_{0}$ and $r_{1}$ ), they get no information about $b$.

Implementing secure channels using oblivious cast. Secure pairwise channels can be achieved by the simulation of authentic channels and the implementation of a pairwise key-agreement protocol between every pair of players $p_{i}$ and $p_{j}$. Players $p_{i}$ and $p_{j}$ can then use the key (e.g., a one-time pad) to encrypt the messages to be sent over the authentic channel.

Lemma 3.2. Let $n \geq 3$. Then authentic channel reduces to oblivious cast for $t<n / 2$.

Proof. An authentic channel between players $p_{i}$ and $p_{j}$ can be achieved from oblivious cast among $p_{i}, p_{j}$, and some arbitrary third player $p_{k} \in P \backslash\left\{p_{i}, p_{j}\right\}$, by $p_{i}$ (or $p_{j}$ ) oblivious-casting his bit (or whole message) $\sigma$ times. Finally, $p_{j}$ decides on the first bit he has received in those oblivious casts.

Since it is sufficient to achieve authentic channels only between pairs of correct players we can assume that the sender is correct. The invocation of this channel fails if $p_{j}$ does not receive any of the bits being sent by oblivious cast, and this happens with a probability of at most $\operatorname{Pr}_{\text {err }}^{\text {auth }}=2^{-\sigma}$.

In order to generate a one-time pad (OTP) $s_{i j}$ of one bit between two players $p_{i}$ and $p_{j}$, we can let $p_{i}$ generate some $m$ random bits $b_{1}, \ldots, b_{m}$ and oblivious-cast them to $p_{j}$ and some arbitrary third player $p_{k}$, where $m$ is chosen such that, with overwhelming probability, $p_{j}$ receives at least one of those random bits (every bit $b_{x}$ is received by $p_{j}$ with probability $\frac{1}{2}$ ). Finally, $p_{j}$ uses his authentic channel to $p_{i}$ (Lemma 3.2) to send to $p_{i}$ the index $x \in\{1, \ldots, m\}$ of the first bit $b_{x}$ that $p_{j}$ received. Since $p_{k}$ gets no 
information about the bit, bit $b_{x}$ can be used as an OTP-bit between $p_{i}$ and $p_{j}$. In order to get an OTP of length $\ell>1$ this process can be repeated $\ell$ times. ${ }^{8}$

In order to guarantee that the transmission of a bit through the secure channel thus obtained fails with an error probability of at most $\operatorname{Pr}_{\text {err }}=2^{-\sigma}$, we can parameterize $m$ and the security parameter for the invocations of the authentic channel, $\sigma_{\text {auth }}$, as follows:

- $\operatorname{Pr}_{\text {err }}^{\mathrm{oc}} \leq 2^{-\sigma-1}$ - the probability that none of the $m$ bits transmitted by oblivious cast is received by player $p_{j}$.

- $\mathrm{Pr}_{\mathrm{err}}^{\text {auth }} \leq 2^{-\sigma-1}$ - the probability that at least one of the invocations of the authentic channel fails.

So we can choose $m=\sigma+1$. The number of invocations of the authentic channel is $\ell=\lceil\log m\rceil+1(\lceil\log m\rceil$ for the transmission of index $x$ plus one for the final transmission of the encrypted bit). Hence, $\sigma_{\text {auth }}$ can be chosen as $\sigma_{\text {auth }}=\sigma+\lceil\log \ell\rceil+1$.

Lemma 3.3. Let $n \geq 3$. Then secure channel reduces to oblivious cast for $t<n / 2$.

Proof. From Lemma 3.2 and the discussion above it follows that the secure channel construction has an error probability of $\operatorname{Pr}_{\text {err }} \leq \operatorname{Pr}_{\text {err }}^{\text {auth }}+\operatorname{Pr}_{\text {err }}^{\text {oc }} \leq 2^{-\sigma}$.

Implementing broadcast using oblivious cast. It is shown in [FM] that a three-party primitive called weak 2-cast, defined below, yields global broadcast secure against $t<n / 2$ active corruptions. Thus, it is sufficient to show that, using oblivious cast, an implementation of weak 2-cast in any set $S \subset P,|S|=3$, and for any selection of a sender among those players, is possible. We first recall the definition of weak 2-cast from $[\mathrm{FM}]$.

Definition 4. Weak 2-cast is a primitive among three players: one sender and two recipients. The sender sends an input bit $b \in\{0,1\}$ and both recipients get an output (decision) value $v \in\{0,1, \perp\}$ such that the following conditions hold:

(1) If both recipients are correct and decide on different values, then one of them decides on $\perp$.

(2) If the sender is correct, then all correct recipients decide on his input bit.

The idea behind the implementation of weak 2-cast using oblivious cast is to have the sender repeatedly oblivious-cast his bit a given number of times. Hence, a recipient who receives two different bits reliably detects that the sender is faulty and may safely decide on $\perp$. On the other hand, in order to make the two recipients decide on different bits, a corrupted sender must oblivious-cast 0's and 1's in such a way that each recipient gets one value, but not the other one. However, since the sender cannot influence which of the recipients gets a bit, he can enforce this situation only with exponentially small probability. We now describe the implementation in more detail.

\footnotetext{
${ }^{8} \mathrm{~A}$ more efficient way to generate an OTP of length $\ell$ is to choose a larger $m$ and have $p_{j}$ send to $p_{i}$ the indices of the first $\ell$ bits he received. For simplicity we restrict ourselves to the less efficient but simpler method.
} 
Protocol Weak-2-Cast-Impl-1 $\left(s,\left\{r_{0}, r_{1}\right\}, \sigma\right)$

1. Sender $s$ oblivious-casts his bit $(\sigma+1)$ times to the recipients.

2. Recipients $r_{i}(i \in\{0,1\})$ decide $v_{i}= \begin{cases}0 & \text { if } 0 \text { received at least once, and no 1's; } \\ 1 & \text { if } 1 \text { received at least once, and no } 0 \text { 's; } \\ \perp & \text { otherwise. }\end{cases}$

Lemma 3.4. Protocol Weak-2-Cast-Impl-1 achieves weak 2-cast with an error probability of at most $2^{-\sigma}$, by only using oblivious cast and local computation.

Proof. If the sender is correct, the protocol can only fail if one of the recipients does not receive any bit from the sender, because the sender always transmits the same bit. This happens with probability $\operatorname{Pr}_{\mathrm{err}_{1}}=2^{-\sigma}$.

If the sender is incorrect, the protocol may fail only if he manages to make one of the recipients receive all 0's and make the other one receive all 1's. In order to achieve this, after having transmitted the first bit, the sender must correctly guess in advance the recipient of every subsequent bit. This happens with probability $\mathrm{Pr}_{\mathrm{err} 2}=2^{-\sigma}$.

Hence, the error probability is $\operatorname{Pr}_{\text {err }} \leq \max \left(\operatorname{Pr}_{\mathrm{err}_{1}}, \mathrm{Pr}_{\mathrm{err}_{2}}\right)=2^{-\sigma}$.

Lemmas 3.2 and 3.4 together with the reduction of broadcast to weak 2-cast and authentic channels in $[\mathrm{FM}]$ immediately yield

Lemma 3.5. Broadcast among $n \geq 3$ players reduces to oblivious cast for $t<n / 2$.

Lemmas 3.3 and 3.5 and the constructions of $[\mathrm{RB}]$ and $\left[\mathrm{CDD}^{+}\right]$yield

Theorem 1. Let $n \geq 3$. Then there is a single-input two-output primitive of cardinality 3 , oblivious cast, that is complete for $t<n / 2$.

\subsubsection{Type [2, 1] Primitives: Converge Cast}

We now show that a cardinality-3 primitive with two inputs and a single output-i.e., the converse of oblivious cast-is also complete for $t<n / 2$.

Definition 5. Converge cast $\left(\mathrm{CC}_{3}\right)$ is a primitive among three players: two senders $s_{0}$ and $s_{1}$ and one recipient $r$. Each sender $s_{i}, i \in\{0,1\}$, sends a value $x_{i}$ from a finite domain $\mathcal{D},|\mathcal{D}| \geq 3$, such that the following conditions hold:

(1) The recipient $r$ receives either $x_{0}$ or $x_{1}$, each with probability $\frac{1}{2}$.

(2) Neither sender learns the other sender's input value, and none of the players learns which of the senders was successful. In case there are other players (apart from $s_{0}, s_{1}$, and $r$ ), they get no information about the input values or the successful sender's identity.

As in the previous section, we show how to implement secure channels and broadcast

(weak 2-cast). We use " $p_{i}, p_{j} \stackrel{?}{\longrightarrow} p_{k}:\left(x_{i}, x_{j}\right)$ " to denote an invocation of converge cast with senders $p_{i}$ and $p_{j}$ sending values $x_{i}$ and $x_{j}$, respectively, and recipient $p_{k}$. 
Furthermore, for two sequences $s_{a}$ and $s_{b}$ of elements in $\{0,1,2\}$ of the same length, we use $\mathcal{H}\left(s_{a}, s_{b}\right)$ to denote the Hamming distance (difference) between the sequences.

Implementing secure channels using converge cast. We now present a protocol to implement a secure channel from $p_{0}$ to $p_{1}$ for the transmission of one bit $x_{0}$. The idea is as follows: first, $p_{1}$ and some other player, say, $p_{2}$, each choose two random keys of an adequate length, one for 0 and for 1 , and converge-cast them to $p_{0} . p_{0}$ stores the two received keys (note that each received key may contain elements from both senders), and uses the corresponding key as input to a converge cast with $p_{1}$ as the recipient to communicate the desired bit. We now describe the protocol in more detail. In what follows, we assume that $\ell$ is a large-enough positive integer.

Protocol Secure-Channel-Impl-2( $\left.p_{0}, p_{1}, \ell\right)$

1. Player $p_{i}, i=1,2$, computes random $\operatorname{keys} s_{i}^{(0)}$ and $s_{i}^{(1)}$ of length $\ell$ over $\{0,1,2\}$

2. $p_{1}, p_{2} \stackrel{\text { ? }}{\longrightarrow} p_{0}:\left(s_{1}^{(0)}, s_{2}^{(0)}\right) ;\left(s_{1}^{(1)}, s_{2}^{(1)}\right) \quad$ (elementwise) $\quad\left(p_{0}\right.$ receives $\left.s_{0}^{(0)} ; s_{0}^{(1)}\right)$

3. $p_{0}, p_{2} \stackrel{?}{\longrightarrow} p_{1}:\left(\sigma_{0}=s_{0}^{\left(x_{0}\right)}, \sigma_{2}=0^{\ell}\right) \quad$ (elementwise; $p_{2}$ always sends 0$)$

$\left(p_{1}\right.$ receives $\left.s_{1}^{\prime}\right)$

4. $p_{1}$ : if $\mathcal{H}\left(s_{1}^{\prime}, s_{1}^{(0)}\right)<\frac{7}{12} \ell$ then $y_{1}=0$, else $y_{1}=1 \mathrm{fi}$

Lemma 3.6. Consider protocol Secure-Channel-Impl-2. For $s \in\{0,1,2\}^{\ell}$ and $k \in\{1, \ldots, \ell\}$ let $s[k]$ denote the projection of $s$ to its $k$ th dimension, i.e., $s=$ $(s[1], \ldots, s[\ell])$. If $p_{0}$ and $p_{1}$ are correct, then for every $k \in\{1, \ldots, \ell\}$,

(1) $s_{1}^{\prime}[k]=s_{1}^{\left(x_{0}\right)}[k]$ with probability $\frac{1}{2}$, and

(2) $s_{1}^{\prime}[k]=s_{1}^{\left(1-x_{0}\right)}[k]$ with probability $\frac{1}{3}$.

Proof. We have to show that this holds independently of $p_{2}$ 's strategy.

(1) We distinguish two cases:

(a) $p_{2}$ enters the same input during steps 2 and $3, x_{2}[k]:=s_{2}^{\left(x_{0}\right)}[k]=\sigma_{2}[k]$. Since $s_{1}^{\left(x_{0}\right)}$ is random (and secret), we have $x_{2}[k]=s_{1}^{\left(x_{0}\right)}[k]$ with probability $P_{1}=\frac{1}{3}$ and $x_{2}[k] \neq s_{1}^{\left(x_{0}\right)}[k]$ with probability $P_{2}=\frac{2}{3}$. Hence the overall probability that $s_{1}^{\prime}[k]=s_{1}^{\left(x_{0}\right)}[k]$ is $P=P_{1} \cdot 1+P_{2} \cdot \frac{1}{4}=\frac{1}{2}$.

(b) $p_{2}$ enters two different inputs during steps 2 and 3, i.e., $s_{2}^{\left(x_{0}\right)}[k] \neq \sigma_{2}[k]$. Since $s_{1}^{\left(x_{0}\right)}$ is random (and secret), we have $s_{1}^{\left(x_{0}\right)}[k] \neq s_{2}^{\left(x_{0}\right)}[k] \wedge s_{1}^{\left(x_{0}\right)}[k] \neq \sigma_{2}[k]$ with probability $P_{1}=\frac{1}{3}$ (in which case $s_{1}^{\prime}[k]=s_{1}^{\left(x_{0}\right)}[k]$ holds with probability $\left.\frac{1}{4}\right) ; s_{1}^{\left(x_{0}\right)}[k]=s_{2}^{\left(x_{0}\right)}[k] \wedge s_{1}^{\left(x_{0}\right)}[k] \neq \sigma_{2}[k]$ with probability $P_{2}=\frac{1}{3}$ (in which case $s_{1}^{\prime}[k]=s_{1}^{\left(x_{0}\right)}[k]$ holds with probability $\left.\frac{1}{2}\right)$; and $s_{1}^{\left(x_{0}\right)}[k] \neq s_{2}^{\left(x_{0}\right)}[k] \wedge$ $s_{1}^{\left(x_{0}\right)}[k]=\sigma_{2}[k]$ with probability $P_{3}=\frac{1}{3}$ (in which case $s_{1}^{\prime}[k]=s_{1}^{\left(x_{0}\right)}[k]$ holds with probability $\left.\frac{3}{4}\right)$. Hence the overall probability that $s_{1}^{\prime}[k]=s_{1}^{\left(x_{0}\right)}[k]$ is $P=P_{1} \cdot \frac{1}{4}+P_{2} \cdot \frac{1}{2}+P_{3} \cdot \frac{3}{4}=\frac{1}{2}$. 
(2) Since $s_{1}^{\left(1-x_{0}\right)}$ is chosen randomly and $p_{2}$ does not get any information about it, the probability that $s_{1}^{\prime}[k]=s_{1}^{\left(1-x_{0}\right)}$ is $P=\frac{1}{3}$.

Lemma 3.7. Let $n \geq 3$. Then secure channel reduces to converge cast for $t<n / 2$.

Proof. Consider protocol Secure-Channels-Impl-2. First, it is easy to see that $p_{2}$ gets no information about bit $x_{0}$. We now show that the channel also provides authenticity. The only ways in which the protocol can fail are that either $x_{0}=0$ and $\mathcal{H}\left(s_{1}^{\prime}, s_{1}^{(0)}\right) \geq \frac{7}{12} \ell$ (call the probability of this event $\operatorname{Pr}_{0}$ ), or that $x_{0}=1$ and $\mathcal{H}\left(s_{1}^{\prime}, s_{1}^{(0)}\right)<$ $\frac{7}{12} \ell$ (probability $\operatorname{Pr}_{1}$ ). These probabilities can be estimated by Chernoff bounds (see the Appendix):

- $\operatorname{Pr}_{0}$ : By Lemma 3.6(1), $s_{1}^{\prime}[k]=s_{1}^{(0)}[k]$ with probability $\frac{1}{2}$. Hence, $\operatorname{Pr}_{0}$ is the probability that out of $N=\ell$ trials with expected value $\mu=\frac{1}{2}$, at most $\frac{5}{12} \ell=\ell / 2-\ell / 12$ are successful. Applying the lower tail bound, we get $\operatorname{Pr}_{0}=\mathcal{C}_{\downarrow}\left(\frac{1}{2}, \ell, \ell / 12-1\right) \leq$ $e^{-2 \varepsilon^{2} / \ell}=e^{-\ell / 72+1 / 3-2 / \ell} \leq e^{-\ell / 72+1}$.

- $\operatorname{Pr}_{1}$ : By Lemma 3.6(2), $s_{1}^{\prime}[k]=s_{1}^{(0)}[k]$ holds with probability $\frac{1}{3}$. Hence, $\operatorname{Pr}_{1}$ is the probability that out of $N=\ell$ trials with expected value $\mu=\frac{1}{3}$, more than $\left(1-\frac{7}{12}\right) \ell=\frac{5}{12} \ell=\ell / 3+\ell / 12$ are successful. Applying the upper tail bound we get $\operatorname{Pr}_{1}=\mathcal{C}_{\uparrow}\left(\frac{1}{3}, \ell, \ell / 12\right) \leq e^{-\ell / 72}$.

Thus, the overall error probability is $\operatorname{Pr}_{\mathrm{err}}^{\text {auth }} \leq \max \left(\operatorname{Pr}_{0}, \operatorname{Pr}_{1}\right)=e^{-\ell / 72+1}$.

Implementing broadcast using converge cast. We now show how weak 2-cast of a bit $x_{0}$ from $p_{0}$ to $p_{1}$ and $p_{2}$ can be simulated using $\mathrm{CC}_{3}$. The idea is as follows: First, $p_{1}$ and $p_{2}$ choose two random keys of an adequate length, one for 0 and for 1 , and converge-cast them to $p_{0} . p_{0}$ stores the two received (mixed) keys. $p_{0}$ then sends his input bit to $p_{1}$ and $p_{2}$ using secure channels. Additionally, $p_{0}$ sends to $p_{1}$ the (received) key corresponding to his input bit. This key can then be used by $p_{1}$ to "prove" to $p_{2}$ which value he received from $p_{0}$. If things "look" consistent to $p_{2}$ (see protocol below), he adopts this value; otherwise, he outputs the value received directly from $p_{0}$. Let " $p_{i} \stackrel{!}{\longrightarrow} p_{j}$ " denote the secure channel from $p_{i}$ to $p_{j}$ (by means of protocol Secure-Channels-Impl-2). The protocol in more detail is as follows:

Protocol Weak-2-Cast-Impl-2( $\left(p_{0},\left\{p_{1}, p_{2}\right\}, \ell\right)$

1. Player $p_{i}, i=1,2$, computes random keys $s_{i}^{(0)}$ and $s_{i}^{(1)}$ of length $2 \ell$ over $\{0,1,2\}$

2. $p_{1}, p_{2} \stackrel{?}{\longrightarrow} p_{0}:\left(s_{1}^{(0)}, s_{2}^{(0)}\right) ;\left(s_{1}^{(1)}, s_{2}^{(1)}\right) \quad$ (elementwise) $\quad\left(p_{0}\right.$ receives $\left.s_{0}^{(0)} ; s_{0}^{(1)}\right)$

3. $p_{0} \stackrel{!}{\longrightarrow} p_{i}(i=1,2): x_{0} \in\{0,1\}$

4. $p_{0} \stackrel{!}{\longrightarrow} p_{1}: s_{0}^{\left(x_{0}\right)}$

$\left(p_{i}\right.$ receives $\left.x_{i} \in\{0,1\}\right)$

5. $p_{1}$ : if $\mathcal{H}\left(s_{1}^{\prime}, s_{1}^{\left(x_{1}\right)}\right) \leq \frac{5}{6} \ell$ then $y_{1}=x_{1}$, else $y_{1}=\perp \mathrm{fi}$

$\left(p_{1}\right.$ receives $\left.s_{1}^{\prime}\right)$

6. $p_{1} \stackrel{\text { ! }}{\longrightarrow} p_{2}: y_{1} ; s_{1}^{\prime}$

$\left(p_{2}\right.$ receives $\left.y_{2} ; s_{2}^{\prime}\right)$

7. $p_{2}$ : if $\left(y_{2}=\perp\right) \vee\left(\mathcal{H}\left(s_{2}^{\prime}, s_{2}^{\left(y_{2}\right)}\right) \geq \frac{7}{6} \ell\right)$ then $y_{2}=x_{2} \mathrm{fi}$ 
Lemma 3.8. Given a pair $(x, y) \in\{0,1,2\}^{2}$ such that $x$ and $y$ are the senders' inputs for an invocation of $\mathrm{CC}_{3}$. If $x$ is chosen uniformly at random from $\{0,1,2\}$, then the receiver's output is $x$ with probability $\operatorname{Pr}_{2}=\frac{2}{3}$.

Proof. By the definition of converge cast, the probability that $x$ is received is $\operatorname{Pr}_{2}=$ $\frac{1}{2} \cdot 1+\frac{1}{2} \cdot \frac{1}{3}=\frac{2}{3}$.

Lemma 3.9. Let $n \geq 3$. Then weak 2 -cast reduces to converge cast for $t<n / 2$.

Proof. Consider protocol Weak-2-Cast-Impl-2. We neglect the error probabilities of the secure channel invocations (protocol Secure-Channels-Impl-2) until the end of the proof. Since the conditions for weak 2-cast are trivially satisfied if more than one player is corrupted, we can distinguish three cases.

All players correct or at most $p_{2}$ corrupted. The only way the protocol can fail is if $p_{1}$ decides on $\perp\left(\mathcal{H}\left(s_{1}^{\prime}, s_{1}^{\left(x_{1}\right)}\right)>\frac{5}{6} \ell\right)$; i.e., that $s_{1}^{\prime}$ and $p_{1}$ 's key differ in more than $\frac{5}{6} \ell$ elements. By Lemma 3.8, since $p_{1}$ is correct, for each invocation of converge cast during step 2 of the protocol it holds that $p_{0}$ does not receive the value $s_{1}^{(*)}$ with probability $1-\operatorname{Pr}_{2}=\frac{1}{3}$. Since all invocations are independent, the failure probability $\operatorname{Pr}_{3}$ can be estimated by a Chernoff bound (see the Appendix) with parameters $\mu=\frac{1}{3}, N=2 \ell$, and $\varepsilon=\frac{5}{6} \ell-\mu N=\frac{5}{6} \ell-\frac{2}{3} \ell=\ell / 6$ :

$$
\operatorname{Pr}_{3} \leq \mathcal{C}_{\uparrow}\left(\frac{1}{3}, 2 \ell, \frac{\ell}{6}\right) \leq e^{-2 \varepsilon^{2} / 2 \ell}=e^{-\ell / 36}
$$

$p_{1}$ corrupted. In order to achieve that $p_{2}$ decides on a wrong output it must hold that $y_{2}=1-x_{0}$ and $\mathcal{H}\left(s_{2}^{\prime}, s_{2}^{\left(y_{2}\right)}\right)<\frac{7}{6} \ell$ before step 7 of the protocol; i.e., that less than $\frac{7}{6} \ell$ elements of $s_{2}^{\prime}$ do not match $p_{2}$ 's key. Since $p_{1}$ does not learn anything about the elements in $s_{2}^{\left(y_{2}\right)}$, he must guess more than $\frac{5}{6} \ell$ out of the $2 \ell$ elements correctly, as otherwise $p_{2}$ would decide on $x_{0}$. For each single element the probability of guessing correctly is $\frac{1}{3}$. Hence the probability of this event can again be estimated by a Chernoff bound with parameters $\mu=\frac{1}{3}, N=2 \ell$, and $\varepsilon=\frac{5}{6} \ell-\mu N=\frac{5}{6} \ell-\frac{2}{3} \ell=\frac{\ell}{6}$ :

$$
\operatorname{Pr}_{4} \leq \mathcal{C}_{\uparrow}\left(\frac{1}{3}, 2 \ell, \frac{\ell}{6}\right) \leq e^{-2 \varepsilon^{2} / 2 \ell}=e^{-\ell / 36} .
$$

$p_{0}$ corrupted. In order to make the protocol fail, $p_{0}$ must select $x_{1} \in\{0,1\}$ and $x_{2}=$ $1-x_{1}$, and achieve that $p_{1}$ decides on $y_{1}=x_{1}$. In this case, since $p_{1}$ and $p_{2}$ are correct, we get $s_{1}^{\prime}=s_{2}^{\prime} \stackrel{\text { def }}{=} s^{\prime}$ and $x_{1}=y_{1}=y_{2} \stackrel{\text { def }}{=} y$ after step 6 . In particular, $p_{0}$ must achieve that $\mathcal{H}\left(s^{\prime}, s_{1}^{(y)}\right) \leq \frac{5}{6} \ell$ and $\mathcal{H}\left(s^{\prime}, s_{2}^{(y)}\right) \geq \frac{7}{6} \ell$, which implies that $s^{\prime}$ must be formed such that it matches $s_{1}^{(y)}$ in at least $\frac{2}{6} \ell=\frac{1}{3} \ell$ more elements than it matches $s_{2}^{(y)}$. For each single element $s^{\prime}[k]$, no matter how $p_{0}$ chooses it, the probability that $s^{\prime}[k]=s_{1}^{(y)}[k] \neq s_{2}^{(y)}[k]$ is the same as the probability that $s^{\prime}[k]=s_{2}^{(y)}[k] \neq s_{1}^{(y)}[k]$, since converge cast is symmetric with respect to $p_{1}$ and $p_{2}$, who both choose their keys uniformly at random. 
Let $X_{k}$ be a random variable that takes on values in $\left\{0, \frac{1}{2}, 1\right\}$, with

$$
X_{k}=\left\{\begin{array}{lll}
0 & \text { if } & s^{\prime}[k]=s_{1}^{(y)}[k] \neq s_{2}^{(y)}[k] \\
\frac{1}{2} & \text { if } & s_{1}^{(y)}[k]=s_{2}^{(y)}[k] ; \quad \text { and } \\
1 & \text { if } & s^{\prime}[k]=s_{2}^{(y)}[k] \neq s_{1}^{(y)}[k]
\end{array}\right.
$$

Then, independently of $p_{0}$ 's strategy, a failure of the protocol corresponds to the probability that for $N=2 \ell$ independent trials with respect to random variable $X_{k}$ (with expected value $\frac{1}{2}$ ), it holds that

$$
\sum_{k=1}^{N=2 \ell} X_{k} \geq \mu N+\frac{1}{2}\left(\frac{7}{6} \ell-\frac{5}{6} \ell\right)=\mu N+\frac{1}{6} \ell .
$$

This probability can be estimated by a Chernoff bound with parameters $\mu=\frac{1}{2}, N=2 \ell$, and $\varepsilon=\ell / 6-1$ :

$$
\operatorname{Pr}_{5} \leq \mathcal{C}_{\uparrow}\left(\frac{1}{2}, 2 \ell, \frac{\ell}{6}-1\right) \leq e^{-2 \varepsilon^{2} / 2 \ell}=e^{-\ell / 36+1 / 3-36 / \ell} \leq e^{-\ell / 36+1} .
$$

Since the error probability of protocol Secure-Channels-Impl-2 can be made negligibly small, it can be parameterized such that the overall probability that at least one invocation fails satisfies $\operatorname{Pr}_{\mathrm{SC}} \leq e^{-\ell / 36}$. Thus, the overall error probability of the weak 2-cast construction based on converge cast is at most

$$
\operatorname{Pr}_{\text {err }} \leq \operatorname{Pr}_{\mathrm{SC}}+\max \left(\operatorname{Pr}_{3}, \operatorname{Pr}_{4}, \operatorname{Pr}_{5}\right) \leq e^{-\ell / 36}+e^{-\ell / 36+1} \leq 2 e^{-\ell / 36+1}<e^{-\ell / 36+2} .
$$

For security parameter $\sigma$, we let $\ell \geq 36(\sigma+2)$, and hence $\operatorname{Pr}_{\text {err }} \leq e^{-\sigma}$.

As before, Lemmas 3.7 and 3.9 and the constructions of $[\mathrm{RB}],\left[\mathrm{CDD}^{+}\right]$, and $[\mathrm{FM}]$ yield

Theorem 2. Let $n \geq 3$. Then there is a two-input, single-output primitive of cardinality 3 , converge cast, that is complete for $t<n / 2$.

We note that allowing the inputs of converge cast to be from a larger domain (than $\{0,1,2\})$ considerably improves the efficiency of our reductions.

\subsubsection{Other Cardinality-3 Primitives}

We note that other primitives of the types considered in this section can be designed with the resources needed to obtain SFE in mind, in such a way that the analysis required by the reductions is less involved. Consider, for example, the following (deterministic) primitive of type [1,2]: The sender $s$ has two input bits, $b_{0}$ (the selection bit) and $b_{1}$ (the actual input), and one of the receivers, say, $r_{0}$, always gets $b_{1}$, regardless of the value of $b_{0}$. If $b_{0}=0$, then $r_{1}$ also gets $b_{1}$; however, if $b_{0}=1$, then $r_{1}$ does not receive anything (alternatively, it gets $\perp$ ). This primitive readily simulates a secure channel (between $s$ 
and $r_{0}$ ), and a 2-cast, but has a more convoluted, less "natural" formulation than oblivious cast, and requires a larger input domain, as well as two instances (an additional instance for secure communication between $s$ and $r_{1}$ ).

Also the cardinality of the input and output domains as well as the internal structure of the primitive are measures that are worth minimizing. One criterion for structural simplicity is symmetry among the senders in the sense that the outputs are invariant under any permutation of the senders' inputs, and symmetry among the recipients in the sense that, for each possible output assignment to the recipients, any permutation of it is also a possible output assignment. Note that oblivious cast and converge cast are both symmetric in this sense and require ternary input and output domains. Asymmetry or non-minimality with respect to the value domains come as a natural trade-off for simpler analysis as mentioned above. For instance, it can be proven that a deterministic primitive of type [1,2] can only be complete for $t<n / 2$ if the cardinality of the input or output domains is larger than 3 , or if the primitive is asymmetric. The main reason for this is that, for a primitive of type $[1,2]$, there must be two different inputs such that one of the recipient's (say, $r_{0}$ 's) output stays invariant while the other recipient's output changes, since otherwise secret communication would be impossible; and, by symmetry, that the same holds with respect to recipient $r_{1}$. However, this implies that broadcast cannot be simulated for the case $n=3$ and $t=1$.

We leave a full characterization of complete primitives with respect to determinism, symmetry, and minimality of the domain sizes as a subject for future research.

\subsection{Primitives of Full Cardinality}

We now introduce the universal black box $\left(\mathrm{UBB}_{n}\right)$, a complete primitive for $t<n$. At first, it might seem trivial to build a complete primitive for arbitrary $t$ by just implementing the functionality of a trusted party. However, computations by trusted parties are generally based on the fact that the trusted party already knows the function to be computed. Since the primitive must be universally applicable, it cannot have any prior information about what is to be computed, i.e., what step of what computation is to be executed. Hence, the specification of the computation step to be performed by the black box must be entered by the players at every invocation of the black box. Although there seems to be no apparent solution to this problem since a dishonest majority might always overrule the honest players' specification, we now describe how the $\mathrm{UBB}_{n}$ effectively overcomes this problem.

For the description, we find it convenient to assume first that the function to be computed has a single output, and present the extension to the general case (multiple outputs, multiple functions) later, i.e., assume that the function to be computed receives inputs from all the players, but exactly one player, say, $p_{0}$, is to learn the result of the computation. (Note that this does not contradict Theorem 7.)

The main idea behind $\mathrm{UBB}_{n}$ for this case is simple: It contains a universal circuit [Va], and has two inputs per player:

- the function input, where the player specifies the function to be computed on all argument inputs, and

- the argument input, where the player inputs his argument to the function. 
The $\mathrm{UBB}_{n}$ now computes the function specified by player $p_{0}$, but for every player that does not input the same function as $p_{0}$, it replaces his argument input by some fixed default value. Finally, the function is computed by evaluating the universal circuit on $p_{0}$ 's function and all argument inputs, and its output is sent to player $p_{0}$. Note that only one invocation of the $\mathrm{UBB}_{n}$ is required. Intuitively, privacy holds since no player other than $p_{0}$ receives any output and therefore learns anything, and $p_{0}$ gets no more information from the other players' arguments than he would from an ideal process involving a trusted party. Correctness follows since the function to be computed is selected by $p_{0}$ himself.

The single-output primitive above can now be turned into a $\mathrm{UBB}_{n}$ to compute multioutput functions by the following modification. Instead of one function, the function input specifies $n$ functions to be computed on the inputs-one function per player. The function $f_{i}, 1 \leq i \leq n$, to be computed and output to player $p_{i}$ is determined by player $p_{i}$ himself, and for the computation of $f_{i}$ the argument inputs of only those players who agree on the same $n$ functions $f_{1}, \ldots, f_{n}$ with $p_{i}$ (i.e., players whose function input matches $p_{i}$ 's) are considered by the $\mathrm{UBB}_{n}$. The following theorem formalizes and extends the argument above.

Theorem 3. For every $n \geq 2$ there is a primitive of cardinality $n$, the $U B B_{n}$, that is complete for $t<n$.

Proof. We argue that privacy and correctness hold for arbitrary $t$.

Privacy: For $1 \leq i \leq n$, a player $p_{i}$ 's output can give information about other player $p_{j}$ 's argument input only if $p_{j}$ entered the same function input as $p_{i}$, which means that $p_{j}$ had "agreed" on exactly this computation. Hence, $p_{i}$ gets the same information about $p_{j}$ 's argument as in an ideal process involving a trusted party. If $p_{i}$ is corrupted and inputs a wrong function input, no argument from a correct player will be used by the $\mathrm{UBB}_{n}$ for this computation.

Correctness: The functions to be computed and output to the correct players are selected by the correct players themselves. Corrupted players that input a different set of functions only achieve that their inputs be replaced by a default value-a strategy that is also (easily) achievable in an ideal process involving a trusted party.

The above has the following implication for computations involving a TTP:

Corollary 3.10 (Oblivious TTPs). Computations involving a TTP do not require the trusted party to have any prior knowledge of the task to be completed by the players.

\subsection{Number of Primitive Instances}

Originally (Section 2.1), we assumed that one primitive instance was available for every permutation of every $k$-player tuple, for a total of $\left(\begin{array}{l}n \\ k\end{array}\right) k$ ! instances. However, as the following theorem shows, fewer instances are required.

Theorem 4. For any $n \geq 2$, each cardinality $k, 2 \leq k \leq n$, and each primitive type, there is a complete primitive such that at most $3\left(\begin{array}{l}n \\ 3\end{array}\right)$ instances are sufficient for unconditional SFE. 
Proof. The theorem follows from Proposition 3.1, the constructions for the proofs of Theorems $1-3$, and from the symmetry of the according primitives.

\section{Impossibility Results}

We first show that the resilience achieved by the primitives given in Sections 3.1 and 3.2 is tight, and that cardinality $k=n$ is necessary for arbitrary resilience; in other words, no primitive of cardinality 2 can do better than $t<n / 3$, no primitive of cardinality 3 can do better than $t<n / 2$, and no primitive of cardinality $k<n$ can do better than $t<n-2$. Then we show that even cardinality $n$ does not help if the primitive is restricted, in the sense of having either a single input (type $[1, *]$ ) or a single output (type $[*, 1])$. Such a primitive is no more powerful than a primitive of cardinality 3 (Section 3.2).

\subsection{Maximal Resilience}

We now prove that no primitive of cardinality $k=2$ can be complete for $t \geq n / 3$ (if $n>2$ ), no primitive of cardinality $k=3$ can be complete for $t \geq n / 2$ (if $n>3$ ), and that, for general $n$, no primitive of cardinality $k=n-1$ can be complete for $t \geq n-2$. This is done by showing that there is a problem, namely broadcast (aka Byzantine agreement) [PSL], that cannot be solved in a model where players are connected by " $g_{k}$ channels," for any $k$-party primitive $g_{k}$. We first recall the definition of broadcast.

Definition 6. Broadcast is a primitive among $n$ players, one sender and $n-1$ recipients. The sender sends an input bit $b \in\{0,1\}$ and the recipients get an output (decision) value $v \in\{0,1\}$ such that the following conditions hold:

Agreement: All correct recipients decide on the same value $v \in\{0,1\}$.

Validity: For all $b \in\{0,1\}$, if the sender is correct and has input $b$, then all correct recipients decide on the sender's input, $v=b$.

In order to give a basic idea of the general impossibility proof (and not to burden the reader with all the counting and parameters required by the general case), we first consider the special case $k=n-1$ and $t \geq n-2$, and later generalize the proof to the case $k \geq 2$ and $t \geq\lceil((k-1) /(k+1)) n\rceil$ (which subsumes the special case), thus covering all the impossibility results announced at the beginning of the section. Both proofs are based on the impossibility proof in [FLM], where it is shown that broadcast for $t \geq\lceil n / 3\rceil$ is not achievable in a model with pairwise authenticated channels. In the new model, however, every subset of $k \geq 2$ players can perform secure multi-party computation. The idea in the proof is to assume that there exists an unconditionally secure broadcast protocol involving all players - interconnected by such " $g_{k}$ channels"-which can then be used to build a different system with contradictory behavior, hence proving that such a protocol cannot exist.

Lemma 4.1. For all $n \geq 2$ and $k<n$, there is no primitive $g_{k}$ that is complete for $t<n$. 
Proof. First note that for every $k$-player primitive with $k<n$, there is an $(n-1)$ player primitive that provides exactly the same functionality by simply ignoring some of the players (see Section 2.1). Hence it is sufficient to show that there is no complete $(n-1)$-player primitive for $t<n$.

Suppose, for the sake of contradiction, that there is an $(n-1)$-player primitive $\mathrm{BB}_{n-1}$ such that broadcast among the $n$ players $p_{0}, \ldots, p_{n-1}$ is reducible to $\mathrm{BB}_{n-1}$ for $t<n-$ and hence also for $t=n-2 .{ }^{9}$ For simplicity, let $\overline{\mathrm{BB}}_{n-1}$ denote the primitive among $n-1$ players that captures (implements) the functionality of all possible instances of $\mathrm{BB}_{n-1}$ that may be shared among $n-1$ players-in general up to $(n-1)$ ! instances, since we allow one instance for each permutation of the players. We now assume that each set of $n-1$ players shares exactly one instance of $\overline{\mathrm{BB}}_{n-1}$.

Let $\Pi=\left\{\pi_{0}, \ldots, \pi_{n-1}\right\}$ denote the players' processors with their local programs and, for each $i \in\{0, \ldots, n-1\}$, let $\pi_{i+n}$ be an identical copy of processor $\pi_{i}$. For every processor $\pi_{k}, k \in\{0, \ldots, 2 n-1\}$, let the number $(k \bmod n)$ be called the type of $p_{k}$.

Instead of connecting the $n$ original processors as required for the broadcast setting, we build a network involving all $2 n$ processors (i.e., the original ones together with their copies) by connecting them with instances of $\overline{\mathrm{BB}}_{n-1}$ such that for every pair of adjacent processors $\pi_{i}$ and $\pi_{(i+1) \bmod 2 n}$ in the new system and without the presence of an adversary, their common view is indistinguishable from their view as two adjacent processors $\pi_{i \bmod n}$ and $\pi_{(i+1) \bmod n}$ in the original system with an adversary that corrupts the processors in $\Pi \backslash\left\{\pi_{i \bmod n}, \pi_{(i+1) \bmod n}\right\}$ in an admissible way.

In order to guarantee that the view of every processor pair $\pi_{i}$ and $\pi_{(i+1) \bmod 2 n}$ is consistent with their view in the original system, the following two conditions must be satisfied:

1. For every processor $\pi_{i}, i \in\{0, \ldots, 2 n-1\}$, and for every selection of $n-2$ processors of types different from $(i \bmod n), \pi_{i}$ shares exactly one $\overline{\mathrm{BB}}_{n-1}$ with these processors (as it does in the original system).

2. If processor $\pi_{i}, i \in\{0, \ldots, 2 n-1\}$, shares an instance of $\overline{\mathrm{BB}}_{n-1}$ with a processor of type $\pi_{(i \pm 1) \bmod n}$ (i.e., an adjacent type in the original system), then it shares it with the concrete processor $\pi_{(i \pm 1) \bmod 2 n}$ (i.e., its adjacent processor of this type in the new system).

This can be achieved by applying the following rule: For each $\overline{\mathrm{BB}}_{n-1}$ that originally connects all processors except $\pi_{i}, i \in\{0, \ldots, n-1\}$, there are now two $\overline{\mathrm{BB}}_{n-1}$ 's, one connecting the processors $\left\{\pi_{i+1}, \ldots, \pi_{i+n-1}\right\}$ and one connecting the processors $\left\{\pi_{(i+1+n) \bmod 2 n}, \ldots, \pi_{(i+n-1+n) \bmod 2 n}\right\}$. This principle is depicted in Fig. 1 for the special case of $n=4$ and $\mathrm{BB}_{3}$.

The new system involves two processors of the type corresponding to the sender, namely, $\pi_{0}$ and $\pi_{n}$, and these are the only processors that enter an input. Let now $\pi_{0}$ and $\pi_{n}$ be initialized with different inputs, i.e., we assume that $\pi_{0}$ has input $v_{0} \in\{0,1\}$ and that $\pi_{n}$ has input $v_{n}=1-v_{0}$.

We now show that there are at least two pairs of adjacent processors in the new system (i.e., a fraction $1 / n$ of all pairs), for which the broadcast conditions are not

\footnotetext{
${ }^{9}$ Since, by definition, broadcast is trivial if strictly less than two players are correct, this is the non-trivial case that involves the least number of correct players.
} 


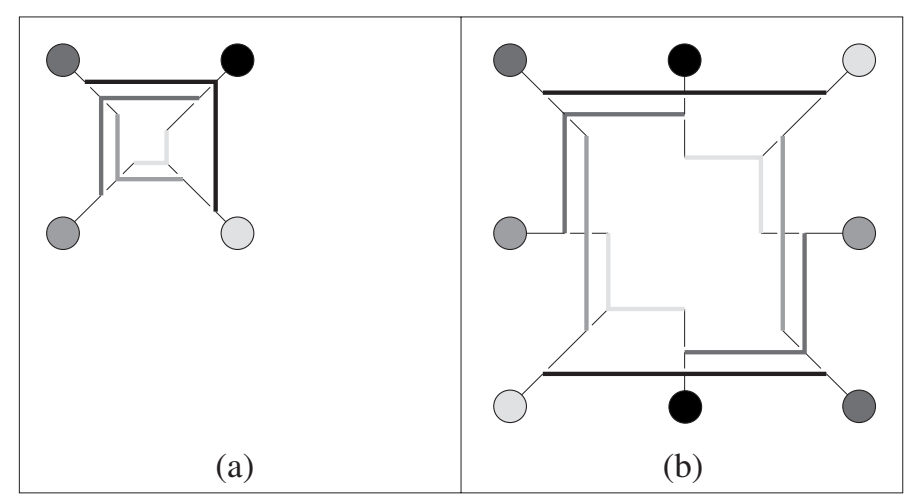

Fig. 1. Reconnection of processors in the proof of Lemma 4.1: special case $n=4$.

satisfied despite being completely consistent with two correct processors in the original system.

First, suppose that the Agreement holds for every pair of processors on, without loss of generality, the value $v_{0}$. Then the Validity condition is violated in both pairs involving processor $\pi_{n}$, since $v_{n} \neq v_{0}$. On the other hand, suppose that the Agreement condition is violated in at least one pair. Then there must exist at least two such pairs because the processors are arranged in a circle. Hence, on inputs $v_{0} \in\{0,1\}$ and $v_{n}=1-v_{0}$, there must be some pair of adjacent processors $(\alpha, \beta)=\left(\pi_{i}, \pi_{(i+1) \bmod 2 n}\right)$ that fails to achieve broadcast with probability at least $1 / n$. Otherwise, strictly less than two pairs would fail per such invocation of the new system. The view of pair $(\alpha, \beta)$ is consistent with the view of the pair $\left(\alpha_{0}, \beta_{0}\right)=\left(\pi_{i}, \pi_{(i+1) \bmod n}\right)$ in the original system for one of the cases where the sender inputs either $v_{0}=0$ or $v_{0}=1$. Hence, in the original system, for some input value $v_{0} \in\{0,1\}$, the adversary can force the pair $\left(\alpha_{0}, \beta_{0}\right)$ to decide on different values with a probability of at least $1 / n$. This contradicts the assumption that broadcast is possible with an arbitrarily small error probability.

Theorem 5. Let $n \geq 3, k<n$, and $t$ be integers. If $t \geq\lceil((k-1) /(k+1)) n\rceil$, then there is no primitive $g_{k}$ complete for $t$.

Proof. As in the proof of Lemma 4.1, let $\overline{\mathrm{BB}}_{k}$ capture the whole functionality of all possible instances of $\mathrm{BB}_{k}$ that may be shared by the same $k$ players.

Suppose, for the sake of contradiction, that for some $k \geq 2$ and $n>k$ there is a broadcast protocol $\mathcal{P}$ for $t \geq\lceil((k-1) /(k+1)) n\rceil$. Let $\Pi=\left\{\pi_{0}, \ldots, \pi_{n-1}\right\}$ be the set of the players' corresponding processors with their local programs and let $\Pi_{0} \dot{\cup} \Pi_{1} \dot{\cup} \ldots \dot{\cup} \Pi_{k}=$ $\Pi$ be a partition of $\Pi$ into $k+1$ sets of cardinalities $\left|\Pi_{i}\right| \in\{\lfloor n /(k+1)\rfloor,\lceil n /(k+1)\rceil\}$. In other words, $\ell=n \bmod (k+1)$ sets of cardinality $\lceil n /(k+1)\rceil$ and $k+1-\ell$ sets of cardinality $\lfloor n /(k+1)\rfloor$, with the additional property that if $\ell \geq(k+1) / 2$, then, of any two adjacent sets $\Pi_{i}$ and $\Pi_{(i+1) \bmod (k+1)}$, at least one is of cardinality $\lceil n /(k+1)\rceil$. Furthermore, for each $i \in\{0, \ldots, n-1\}$, let $\pi_{i+n}$ be an identical copy of processor $\pi_{i}$. Finally, for each $i \in\{0, \ldots, k\}$, let $\Pi_{i+n}=\left\{\pi_{k+n} \mid \pi_{k} \in \Pi_{i}\right\}$ form an identical copy of set $\Pi_{i}$. 
We now proceed as in the proof of Lemma 4.1. Instead of connecting the original processors as required for the broadcast setting, we build a network involving all $2 n$ processors by connecting them with instances of $\overline{\mathrm{BB}}_{k}$ such that for every set $\Pi_{i} \cup$ $\Pi_{(i+1) \bmod (2 k+2)}$ of processors in the new system, and without the presence of an adversary, their common view is indistinguishable from their view as processors in $\Pi_{i \bmod (k+1)} \cup$ $\Pi_{(i+1) \bmod (k+1)}$ in the original system with an adversary that corrupts the processors in $\Pi \backslash\left(\Pi_{i \bmod (k+1)} \cup \Pi_{(i+1) \bmod (k+1)}\right)$ in an admissible way.

Note that for every $\overline{\mathrm{BB}}_{k}$ in the original system there is a set $\Pi_{i}$ such that no processor in $\Pi_{i}$ is connected to it. This is because there are $k+1$ different sets $\Pi_{i}$. The $\overline{\mathrm{BB}}_{k}$ 's are now reconnected in the following way: For each $\overline{\mathrm{BB}}_{k}$ that originally connects a set $S$ of $k$ processors in $\Pi \backslash \Pi_{i}(i \in\{0, \ldots, k\})$, there are now two $\overline{\mathrm{BB}}_{k}$ 's, one connecting processors $\left\{\pi_{j} \in \Pi_{i+1} \cup \ldots \cup \Pi_{i+n-1} \mid \pi_{j \bmod (k+1)} \in S\right\}$ and one connecting processors $\left\{\pi_{j} \in \Pi_{i+1+n} \cup \ldots \cup \Pi_{i+n-1+n} \mid \pi_{j \bmod (k+1)} \in S\right\}$.

We now show that every set $\Pi_{i} \cup \Pi_{(i+1) \bmod (2 k+2)}$ of processors contains at least $n-t$ processors, implying that protocol $\mathcal{P}$ satisfies the broadcast conditions with respect to all processors in the union of two such adjacent processor sets. We have two cases:

$$
\begin{aligned}
& \ell<\frac{k+1}{2}: \\
& \begin{aligned}
\left|\Pi_{i} \cup \Pi_{(i+1) \bmod (2 k+2)}\right| & \geq 2\left\lfloor\frac{n}{k+1}\right\rfloor=\left\lfloor\frac{2 n}{k+1}\right\rfloor=n+\left\lfloor\frac{2 n-(k+1) n}{k+1}\right\rfloor \\
& =n-\left\lfloor\frac{k-1}{k+1} n\right\rfloor \geq n-\left\lceil\frac{k-1}{k+1} n\right\rfloor \geq n-t ;
\end{aligned}
\end{aligned}
$$

and

$$
\ell \geq \frac{k+1}{2}: \quad\left|\Pi_{i} \cup \Pi_{(i+1) \bmod (2 k+2)}\right| \geq\left\lfloor\frac{n}{k+1}\right\rfloor+\left\lceil\frac{n}{k+1}\right\rceil \geq\left\lfloor\frac{2 n}{k+1}\right\rfloor \geq n-t .
$$

The proof now proceeds in exactly the same way as the proof of Lemma 4.1. By assigning different input values to both senders, $\pi_{0}$ and $\pi_{n}$, we obtain a non-negligible error probability of at least $1 /(k+1)$, which contradicts the assumption of broadcast being possible with an arbitrarily small error probability.

The following corollary makes the relevant cases explicit:

\section{Corollary 4.2.}

- Let $n \geq 3$. Then there is no primitive $g_{2}$ complete for $t \geq\lceil n / 3\rceil$.

- Let $n \geq 4$. Then there is no primitive $g_{3}$ complete for $t \geq\lceil n / 2\rceil$.

- Let $n>k$. Then there is no primitive $g_{k}$ complete for $t \geq n-2$.

Moreover, there is some evidence that no primitive of cardinality $k<n$ can be complete even for $t \geq\lceil n / 2\rceil$ : Since $k<n$, then, evidently, there are computations (e.g., the logical AND over all players' inputs) that would require more than one primitive invocation, but, on the other hand, secret sharing is not possible if $t \geq\lceil n / 2\rceil$ and thus it seems impossible to securely convey non-trivial output information from one primitive invocation to the input of the next one. So we put forward the following: 
Conjecture 4.3. For all $n \geq 2$ and any $k<n$, there is no primitive $g_{k}$ complete for $t \geq\lceil n / 2\rceil$.

\subsection{Limitation of Multicast and Convergecast}

We now take a closer look at primitives that are restricted to either one single input (i.e., type $[1, *]$, or "multicast") or one single output (i.e., type $[*, 1])$, or "convergecast"). We show that any such primitive, even with full cardinality $(k=n)$, cannot be complete for any $t \geq\lceil n / 2\rceil$, implying that the examples for primitives of types $[1,2]$ and $[2,1]$ given in Section 3.2 are as powerful as any primitive of type $[1, n]$ or $[n, 1]$. Since, for $k \leq n$, any primitive of type $[1, k]$ (or $[k, 1]$ ) can be simulated by a primitive of type $[1, n]$ (or $[n, 1]$, respectively), it is sufficient to show the impossibility for primitives of type $[1, n]$ (or $[n, 1])$.

Theorem 6. For all $n \geq 2$, there is no primitive of type $[1, n]$ complete for $t \geq\lceil n / 2\rceil$.

Proof. The proof is by contradiction. Assume that there exists a primitive of type $[1, n]$, $\mathrm{M}_{n}$, that is complete for multi-party computation among players $P=\left\{p_{1}, \ldots, p_{n}\right\}$ for $t \geq\lceil n / 2\rceil$. Then, in particular, for every function $f$ there is a protocol $\Pi_{f}$ which allows the players in $P$ to compute this function privately in the presence of up to $t$ passively corrupted players, and which only makes use of $\mathbf{M}_{n}$.

We now show that $\Pi_{f}$ can be simulated in the standard model with secure pairwise channels. Since each invocation of $\mathbf{M}_{n}$ involves the input of at most one player, say input $x_{i}$ from player $p_{i}$, and produces outputs, call them $m_{1}\left(x_{i}\right), \ldots, m_{n}\left(x_{i}\right)$, one for each player in $P$, this invocation can be simulated in the standard model by player $p_{i}$ himself computing each output $m_{j}\left(x_{i}\right), 1 \leq j \leq n$, and secretly sending them to each player.

As a result, in the secure channels model, the players in $P$ can securely compute any function in the presence of a passive adversary that corrupts $t \geq\lceil n / 2\rceil$ of the players. However, as shown in [BGW], this is not possible.

We now show a similar result for convergecast primitives of arbitrary cardinality.

Theorem 7. For all $n \geq 2$, there is no primitive of type $[n, 1]$ complete for $t \geq\lceil n / 2\rceil$.

Proof. The proof is by reduction to a two-party problem known to have no solution. Assume that there exists a primitive of type $[n, 1], \mathrm{C}_{n}$, that is complete for multi-party computation among players $P=\left\{p_{1}, \ldots, p_{n}\right\}$ for $t \geq\lceil n / 2\rceil$. We show that the existence of $\mathrm{C}_{n}$ implies the existence of a complete primitive of type [2,1] for secure two-party computation where both parties learn the result of the computation.

For this, consider some function $f\left(x_{1}, x_{2}\right)$ (i.e., a function of players $p_{1}$ and $p_{2}$ 's inputs) that is to be computed by players in $P$, and let $\Pi_{f}$ denote the protocol that allows all correct players to learn $f\left(x_{1}, x_{2}\right)$ in the presence of $t \geq\lceil n / 2\rceil$ actively corrupted players, while only making use of primitive $\mathrm{C}_{n}$. Consider now the two-party setting, with players $q_{1}$ and $q_{2}$, one of which might be actively corrupted. We show how, based on the assumed solution for the multi-party setting, $q_{1}$ and $q_{2}$ are able to compute $f$. 
The construction goes as follows. We partition the set $P$ into two sets, $P_{1}$ and $P_{2}$, such that $\left|P_{1}\right|=t,\left|P_{2}\right|=n-t(\leq t)$, and with $p_{1} \in P_{1}$ and $p_{2} \in P_{2}$. Player $q_{1}$ simulates all players in $P_{1}$ with $p_{1}$ 's input as his own input, and player $q_{2}$ simulates all players in $Q_{2}$ with $p_{2}$ 's input as his input. Furthermore, every input and output of each $\mathrm{C}_{n}$ primitive used in $P$ is reconnected to the respective simulating player, $q_{1}$ and $q_{2}$; as a result, each instance of $\mathrm{C}_{n}$ in the original setting can be replaced by a primitive of type $[2,1]$. By assumption, since each player simulates at most $t$ players in $P$, the resulting protocol securely computes and delivers output $f\left(x_{1}, x_{2}\right)$ to both players.

Thus, there is a two-player primitive with a single output that is complete for secure two-party computation where both players learn the same result. This is a direct contradiction to the "one-sidedness" observation in [BMM], that a protocol based on an asymmetric (i.e., single-output) two-player primitive cannot guarantee that both players learn the result of the computation.

\section{Summary and Open Problems}

In this paper we have put forward the concept of minimal cardinality of primitives that are complete for SFE. Since this is a new line of research, several questions remain open.

We completely characterized the cases of types $[1,1],[1, k]$, and $[k, 1]$, for all cardinalities $k \leq n$. In particular, for $t<n / 3$ there is a complete primitive, $\mathrm{SC}_{2}[1,1]$, and no $g_{2}$ can do any better; and, for $t<n / 2$, there are two complete primitives, $\mathrm{OC}_{3}[1,2]$ and $\mathrm{CC}_{3}[2,1]$, and no $g_{k}, k \leq n$, can do any better. For the case of type $[k, k]$ it remains to prove Conjecture 4.3, that no $g_{n-1}[n-1, n-1]$ is complete for $t \geq\lceil n / 2\rceil$. This would partition the whole hierarchy into three equivalence classes of cardinalities $k=2$ $(t<n / 3), 2<k<n(t<n / 2)$, and $k=n(t<n)$.

It would also be interesting to analyze the completeness of primitives as a function of the size of the input and output domains. We took some initial steps for the case of cardinality 3 in Section 3.2.3. Also the completeness of the $\mathrm{UBB}_{n}$ for $t<n$ relies on the fact that inputs of large size are allowed.

\section{Acknowledgments}

We thank the anonymous referees for their many valuable comments; in particular, the example of an asymmetric deterministic primitive in Section 3.2.3 was suggested by one of the referees. The work of Matthias Fitzi was partly done while visiting Bell Labs and DIMACS.

\section{Appendix. Chernoff Bounds}

In the analysis of our protocols we apply Chernoff bounds in order to estimate upper bounds on their error probabilities. We present a succinct description of these bounds here for convenience.

Let $X_{i} \in[0,1](1 \leq i \leq N)$ be a sequence of independent random variables with expected value $\mu$. The Chernoff bound gives an upper bound on the probability that the 
sum $\sum_{i=1}^{N} X_{i}$ deviates from the expected value $\mu N$ by a given distance $\varepsilon>0$ :

$$
\begin{aligned}
& \mathcal{C}_{\downarrow}(\mu, N, \varepsilon)=\operatorname{Prob}\left(\sum_{i=1}^{N} X_{i}<\mu N-\varepsilon\right) \leq e^{-2 \varepsilon^{2} / N}, \\
& \mathcal{C}_{\uparrow}(\mu, N, \varepsilon)=\operatorname{Prob}\left(\sum_{i=1}^{N} X_{i}>\mu N+\varepsilon\right) \leq e^{-2 \varepsilon^{2} / N} .
\end{aligned}
$$

\section{References}

[Be] D. Beaver. Foundations of secure interactive computation. In Advances in Cryptology - CRYPTO '91, LNCS, pp. 377-391. Springer-Verlag, Berlin, 1992.

[BGW] M. Ben-Or, S. Goldwasser, and A. Wigderson. Completeness theorems for non-cryptographic faulttolerant distributed computation. In Proc. 20th ACM Symp. on the Theory of Computing, pp. 1-10, 1988.

[B1] M. Blaze. Oblivious key escrow. In R. Anderson, editor, Proc. First Infohiding, volume 1174 of LNCS, pp. 335-343, Springer-Verlag, Berlin, 1996.

[BMM] A. Beimel, T. Malkin, and S. Micali. The all-or-nothing nature of two-party secure computation. In Advances in Cryptology - CRYPTO '99, volume 1666 of LNCS, pp. 80-97. Springer-Verlag, Berlin, 1999.

[Ca] R. Canetti. Security and composition of multiparty cryptographic protocols. Journal of Cryptology, 13(1):143-202, 2000.

[CCD] D. Chaum, C. Crépeau, and I. Damgård. Multiparty unconditionally secure protocols (extended abstract). In Proc. 20th ACM Symp. on the Theory of Computing, pp. 11-19, 1988.

$\left[\mathrm{CDD}^{+}\right]$R. Cramer, I. Damgård, S. Dziembowski, M. Hirt, and T. Rabin. Efficient multiparty computations secure against an adaptive adversary. In Advances in Cryptology - EUROCRYPT '99, volume 1592 of LNCS, pp. 311-326. Springer-Verlag, Berlin, 1999.

[Ch] D. Chaum. The dining cryptographers problem: unconditional sender and recipient untraceability. Journal of Cryptology, 1(1):65-75, 1988.

[DDWY] D. Dolev, C. Dwork, O. Waarts, and M. Yung. Perfectly secure message transmission. Journal of the ACM, 40(1):17-47, Jan. 1993.

[FLM] M. J. Fischer, N. A. Lynch, and M. Merritt. Easy impossibility proofs for distributed consensus problems. Distributed Computing, 1:26-39, 1986.

[FM] M. Fitzi and U. Maurer. From partial consistency to global broadcast. In Proc. 32nd Annual Symp. on the Theory of Computing, pp. 494-503, 2000.

[GL] S. Goldwasser and L. Levin. Fair computation of general functions in presence of immoral majority. In Advances in Cryptology - CRYPTO '90, volume 537 of LNCS, pp. 77-93. Springer-Verlag, Berlin, 1990.

[GMW] O. Goldreich, S. Micali, and A. Wigderson. How to play any mental game. In Proc. 19th ACM Symp. on the Theory of Computing, pp. 218-229, 1987.

[Go1] O. Goldreich. Preface. Journal of Cryptology, Special issue on General Secure Multiparty Computation, 13(1):1-8, 2000.

[Go2] O. Goldreich. Secure multi-party computation, Working draft, version 1.2, Mar. 2000.

[Ki1] J. Kilian. Founding cryptography on oblivious transfer. In Proc. 20th Annual ACM Symp. on the Theory of Computing, pp. 20-31, 1988.

[Ki2] J. Kilian. A general completeness theorem for two-party games. In Proc. 23rd Annual ACM Symp. on the Theory of Computing, pp. 553-560, 1991.

[Ki3] J. Kilian. More general completeness theorems for secure two-party computation. In Proc. 32nd Annual ACM Symp. on the Theory of Computing, pp. 316-324, 2000.

[KKMO] J. Kilian, E. Kushilevitz, S. Micali, and R. Ostrovsky. Reducibility and completeness in private computations. SIAM Journal on Computing, 29:1189-1208, 1999. 
[KMO] E. Kushilevitz, S. Micali, and R. Ostrovsky. Reducibility and completeness in multi-party private computations. In Proc. 35th Annual IEEE Symp. on the Foundations of Computer Science, pp. 478-491, 1994.

[MR] S. Micali and P. Rogaway. Secure computation. In Advances in Cryptology - CRYPTO '91, volume 576 of LNCS, pp. 392-404. Springer-Verlag, Berlin, 1992.

[PSL] M. Pease, R. Shostak, and L. Lamport. Reaching agreement in the presence of faults. Journal of the ACM, 27(2):228-234, Apr. 1980.

[Ra] M. O. Rabin. How to exchange secrets by oblivious transfer. Technical Report TR-81, Harvard Aiken Computation Laboratory, 1981.

[RB] T. Rabin and M. Ben-Or. Verifiable secret sharing and multiparty protocols with honest majority. In Proc. 21st ACM Symp. on the Theory of Computing, pp. 73-85, 1989.

[Va] L. G. Valiant. Universal circuits. In Proc. ACM Symp. on Theory of Computing (STOC '76), pp. 196-203. ACM Press, New York, 1976.

[Ya] A. C. Yao. Protocols for secure computations. In Proc. 23rd IEEE Symp. on the Foundations of Computer Science, pp. 160-164, 1982. 